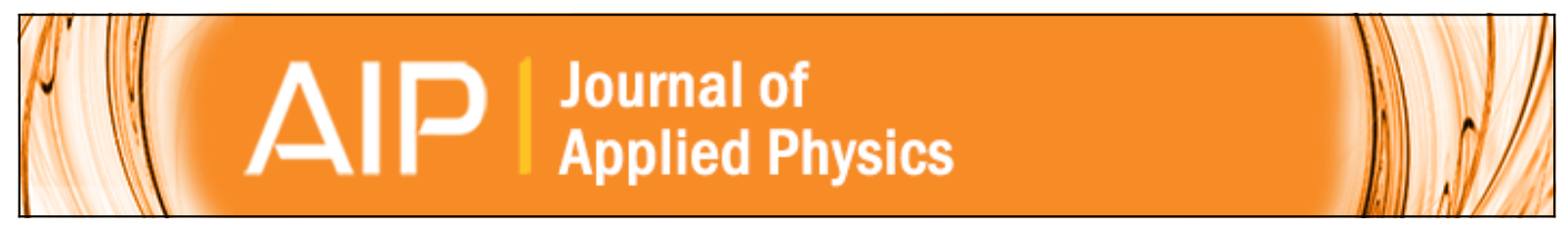

Dependence of the magnetization and remanence of single-domain particles on the second cubic anisotropy constant

J. Geshev, L. G. Pereira, J. E. Schmidt, and M. Mikhov

Citation: Journal of Applied Physics 90, 6243 (2001); doi: 10.1063/1.1415062

View online: http://dx.doi.org/10.1063/1.1415062

View Table of Contents: http://scitation.aip.org/content/aip/journal/jap/90/12?ver=pdfcov

Published by the AIP Publishing 


\title{
Dependence of the magnetization and remanence of single-domain particles on the second cubic anisotropy constant
}

\author{
J. Geshev, ${ }^{\text {a) }}$ L. G. Pereira, and J. E. Schmidt \\ Instituto de Física, Universidade Federal do Rio Grande do Sul, CP 15051, 91501-970, Porto Alegre, \\ RS, Brazil \\ M. Mikhov \\ Faculty of Physics, "St. Kliment Ohridski" University of Sofia, 1126 Sofia, Bulgaria
}

(Received 30 November 2000; accepted for publication 2 September 2001)

\begin{abstract}
Magnetization and remanent magnetization curves for noninteracting single-domain particles with cubic magnetocrystalline anisotropy have been calculated, taking into account the first two anisotropy constants. The dependencies of the saturation remanence, coercivity, remanence coercivity, and $\delta M$ plots on the anisotropy constants ratio have been discussed. It has been found that the calculated saturation remanence shows maxima in the vicinity of the spin-reorientation transition points for both negative and positive first cubic anisotropy constants. The remanent magnetization for the case when the face diagonals $\langle 110\rangle$ are the easiest magnetization orientations has been analytically determined as well. It has been shown that when more than one type of easiest directions coexist, the remanence of the system can only be obtained numerically because of the history dependence of the remanent magnetization for some particles' configurations. The predicted remanence behavior for systems representing spin-reorientation has been compared with that of a $\mathrm{Tb}_{0.6} \mathrm{Ho}_{0.4} \mathrm{Fe}_{2}$ powder sample, and an excellent agreement between numerical and experimental data has been found. (C) 2001 American Institute of Physics. [DOI: 10.1063/1.1415062]
\end{abstract}

\section{INTRODUCTION}

Nanostructured materials at present attract much attention because of the wide range of their actual and potential technological applications. Among them are magnetic recording, ferrofluids, catalysts, color imaging, and pigments in paints and ceramics.

The calculation of the hysteresis behavior of such systems has been subjected to considerable attention over a period of many years. The first systematic model studies were performed for an assembly of uniaxial single-domain particles in 1948 by Stoner and Wohlfarth ${ }^{1}$ and subsequently a lot of papers have been published on this subject. The predicted remanent magnetization, $M_{r}$, for a random assembly of such uniaxial anisotropy particles is equal to $0.5 M_{s}$, where $M_{s}$ is the saturation magnetization.

Materials with multiaxial cubic or competing types of magnetic anisotropy have been widely studied experimentally and, to a lesser extent, theoretically. Partial magnetization curve calculations for the case of cubic magnetocrystalline anisotropy have been done in 1961 by Johnson and Brown ${ }^{2}$ and in 1966 by Lee and Bishop. ${ }^{3}$ The series expansions of Gans, ${ }^{4}$ the integration of Kaya, ${ }^{5}$ and the interpolation of Stoner and Rhodes ${ }^{6}$ give an $M_{r}$ of $0.8312 M_{s}$ and $0.8660 M_{s}$ for positive and negative first-order magnetocrystalline anisotropy constant $K_{1}$, respectively. Whole hysteresis loops were not determined by these authors since it was revealed that for a cubic anisotropy particle, though it is always possible to calculate the critical orientation and the critical magnetic field at which a certain magnetization state

${ }^{a)}$ Electronic mail: julian@if.ufrgs.br loses its stability and a discontinuous jump of the magnetization starts, there may be available several stable magnetization positions where this jump may terminate. Thus, strictly speaking, it is impossible to obtain a complete hysteresis loop for an individual particle., ${ }^{2,3}$ Fortunately, the shape of the hysteresis loop and the coercivity of random particle assembly with cubic anisotropy are almost independent on the precise sequence of irreversible rotations which occur during the reversal process, as shown by Usov and Peschany.

The first complete hysteresis loops have been calculated in 1974 by Joffe and Heuberger. ${ }^{8}$ Most recently, Geshev et al. ${ }^{9,10}$ have reported theoretical predictions on the thermomagnetic and remanence curves of such systems, and Walker et al. ${ }^{11,12}$ extended the work of Joffe and Heuberger by taking into account the effects of thermally activated magnetization reversal on the magnetization and remanent magnetization curves.

Theoretical results have also been obtained for the remanent magnetization of single-domain particles having mixed uniaxial anisotropies by Wohlfarth and Tonge; ${ }^{13}$ several cases of anisotropy made up of cubic magnetocrystalline and uniaxial components have been investigated by Tonge and Wohlfarth, ${ }^{14}$ Arrott, ${ }^{15}$ and Geshev et al. ${ }^{16,17}$

For most of the cubic anisotropy materials, the first two anisotropy constants $K_{1}$ and $K_{2}$ are comparable in magnitude, and at room temperature usually $K_{1}>K_{2}$. Even when $K_{1}=K_{2}$, the contribution of the term containing $K_{2}$ to the difference between maximum and minimum anisotropy energy is only $1 / 9$ of the contribution of the term containing $K_{1}$, i.e., in most of the cases the term containing $K_{2}$ is small 
TABLE I. Directions of easiest, intermediate, and hardest magnetization, and the corresponding anisotropy fields for cubic anisotropy.

\begin{tabular}{|c|c|c|c|c|c|c|}
\hline $\begin{array}{l}K_{1} \\
K_{2}\end{array}$ & $\begin{array}{c}+ \\
+\infty \text { to } \\
-9\left|K_{1}\right| / 4\end{array}$ & $\begin{array}{c}+ \\
-9 K_{1} / 4 \\
\text { to }-9 K_{1}\end{array}$ & $\begin{array}{c}+ \\
-9 K_{1} \\
\text { to }-\infty\end{array}$ & $\begin{array}{c}- \\
-\infty \text { to } \\
9\left|K_{1}\right| / 4 \\
\end{array}$ & $\begin{array}{c}- \\
9\left|K_{1}\right| / 4 \\
\text { to } 9\left|K_{1}\right|\end{array}$ & $\begin{array}{c}- \\
9\left|K_{1}\right| \\
\text { to }+\infty\end{array}$ \\
\hline Easiest & 100 & 100 & 111 & 111 & 110 & 110 \\
\hline Intermediate & 110 & 111 & 100 & 110 & 111 & 100 \\
\hline Hardest & 111 & 110 & 110 & 100 & 100 & 111 \\
\hline Anisotropy field & \multicolumn{2}{|c|}{$2 K_{1} / M_{s}$} & \multicolumn{2}{|c|}{$\frac{4}{3}\left(K_{1}+\frac{1}{3} K_{2}\right) / M_{s}$} & \multicolumn{2}{|c|}{$\left(K_{1}+\frac{1}{2} K_{2}\right) / M_{s}$} \\
\hline
\end{tabular}

compared to the one containing $K_{1}$. However, for magnetization reversal in some specific crystallographic planes, the contribution of the term containing $K_{2}$ could not be neglected. For example, if the rotation of the magnetization vector takes place in the [111] plane, the $K_{1}$ term is a constant and the anisotropy is determined by the higher order energy terms.

Even when the $K_{1}$ term is not constant, the influence of $K_{2}$ on the magnetization of a material can be crucial. Both $K_{1}$ and $K_{2}$ are temperature dependent, $K_{1}$ can pass through zero at a certain temperature, so the $\left|K_{2} / K_{1}\right|$ ratio can be very high in some temperature range; in such cases one must take into account the term containing $K_{2}$ in the energy expression in order to explain the magnetic behavior of the material. Rare-earth iron garnets ${ }^{18}$ and rare-earth iron ternary cubic Laves phase compounds ${ }^{19-21}$ are examples of such magnetic systems. Some numerical results on the influence of $K_{2}$ on the hysteresis loops of noninteracting single domain particles have been reported by Usov and Peschany ${ }^{7}$ and by García-Otero et al. ${ }^{22}$

In this article, we present detailed model calculations of the magnetization and remanence curves for a disordered system of noninteracting single-domain particles with cubic anisotropy, taking into consideration both the first and the second anisotropy constants. The thermal activation effects have been neglected and only coherent rotations of the magnetization have been considered. The magnetization calculations cover a much wider $K_{2}$ range than the previous ones. The predicted remanence behavior for systems representing spin-reorientation was compared to the one of a $\mathrm{Tb}_{0.6} \mathrm{Ho}_{0.4} \mathrm{Fe}_{2}$ fine powder sample.

\section{MODEL}

It is well established that in a cubic crystal the energy $E_{a}$ of magnetization per unit volume can be expressed as a function of the direction cosines $\alpha_{1}, \alpha_{2}$, and $\alpha_{3}$ of the magnetization vector $\mathbf{M}_{s}$, referred to the cube axes. In ascending powers of the direction cosines, the first two nonconstant terms of the energy expression are

$$
E_{a}=K_{1}\left(\alpha_{1}^{2} \alpha_{2}^{2}+\alpha_{2}^{2} \alpha_{3}^{2}+\alpha_{3}^{2} \alpha_{1}^{2}\right)+K_{2} \alpha_{1}^{2} \alpha_{2}^{2} \alpha_{3}^{2} .
$$

It is possible to analytically determine which are the easiest magnetization directions. Using only the $K_{1}$ term of Eq. (1), when $K_{1}>0$ there are six energy minima along the principal cube axes, i.e., the $\langle 100\rangle$ directions, and eight maxima along the body diagonals $\langle 111\rangle$. When $K_{1}<0$, the maxima and minima orientations obtained for $K_{1}>0$ are in- terchanged. There are eight easiest directions of the type $\langle 111\rangle$ and six hardest directions of the type $\langle 100\rangle$.

When both $K_{1}$ and $K_{2}$ terms are important, $E_{a}$ may also be a minimum for $\langle 110\rangle$. The directions of easiest, intermediate, and hardest magnetization for all combinations of values of $K_{1}$ and $K_{2}$, first calculated by Bozorth, ${ }^{23}$ are given in Table I. It can be seen that the easiest, intermediate, and hardest directions may have any permutation of the order $\langle 100\rangle,\langle 110\rangle$, and $\langle 111\rangle$.

When a spherical single-domain particle with cubic anisotropy is placed in an external magnetic field $\mathbf{H}$, the total free energy of this particle in units of $2\left|K_{1}\right|$ is

$$
\begin{aligned}
\eta= & \frac{1}{2} \operatorname{sgn}\left(K_{1}\right)\left(\alpha_{1}^{2} \alpha_{2}^{2}+\alpha_{2}^{2} \alpha_{3}^{2}+\alpha_{3}^{2} \alpha_{1}^{2}\right)+\frac{K_{2}}{2\left|K_{1}\right|} \alpha_{1}^{2} \alpha_{2}^{2} \alpha_{3}^{2} \\
& -h \cos \varphi .
\end{aligned}
$$

Here $h$ is the reduced magnetic field $\left(=H M_{s} / 2\left|K_{1}\right|\right)$ and $\cos \varphi$ is given by

$$
\cos \varphi=\cos \gamma \cos \theta+\sin \gamma \sin \theta \cos (\vartheta-\psi),
$$

where $\gamma$ and $\vartheta$ are the polar and the azimuthal angles of $\mathbf{M}_{s}$, and $\theta$ and $\psi$ are those of $\mathbf{H} ; \varphi$ is the angle between $\mathbf{M}_{s}$ and H.

It is difficult to find the minima of the energy function when $H \neq 0$ and, in general, this can only be done numerically. In the present work, magnetization $M(H)$ and remanence $M_{r}(H)$ and $M_{d}(H)$ curves for a disordered system of noninteracting single-domain particles with cubic anisotropy were numerically calculated from Eq. (2) by finding the angles $\gamma$ and $\vartheta$ for which $\eta$ is at minimum. For the hysteresis loop calculations the magnetic field was continuously varied between $H_{\max }$ and $-H_{\max }$, where $H_{\max }$ is higher than the corresponding anisotropy field $H_{a}$. The two-variable minimization procedure used is described elsewhere. ${ }^{16,17}$ The $\mathrm{dc}$ demagnetization remanence curve $M_{d}(H)$ is obtained by first saturating the sample in a positive field and then measuring the remanence $M_{d}$ after application and removal of negative fields. ${ }^{24}$ The isothermal remanent magnetization curve $M_{r}(H)$ is instead obtained by applying and removing the field $H$ to the demagnetized state; both curves are normalized to $M_{r}(\infty)$.

\section{RESULTS AND DISCUSSION}

\section{A. Model hysteresis loops}

Several representative hysteresis loops calculated for negative and positive $K_{1}$ are shown in Figs. 1(a) and 1(b), 


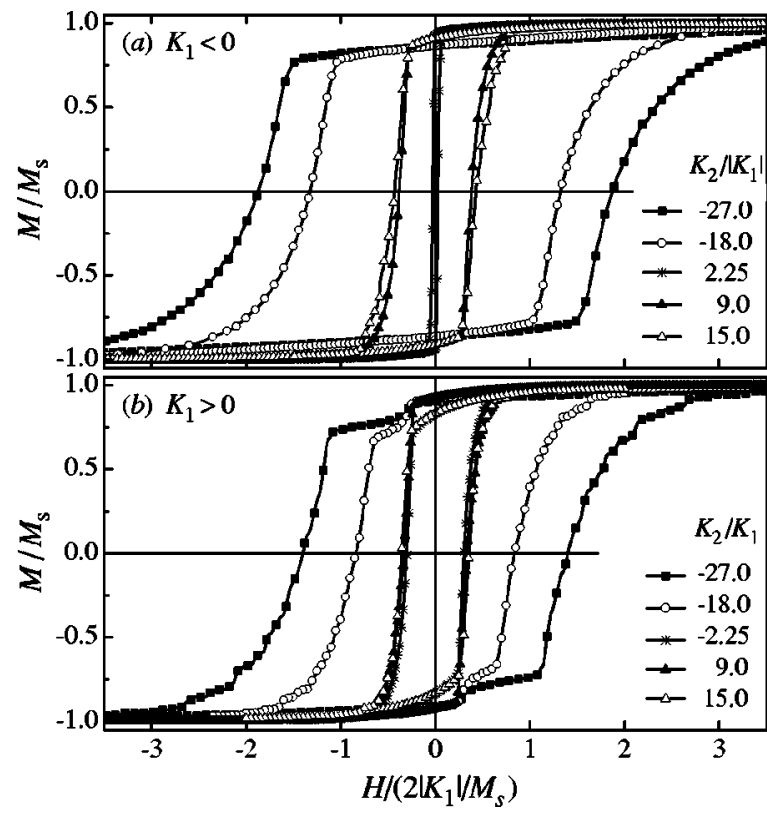

FIG. 1. Representative model hysteresis loops for negative (a) and positive (b) $K_{1}$ and different $K_{2} /\left|K_{1}\right|$ ratios.

respectively. It is seen that the saturation remanence, the coercive field, as well as the shape of the loops are influenced by the $K_{2}$ value. Although the influence is weaker for $K_{1}$ $>0$, which is in accordance with previous calculations, ${ }^{7,22}$ steps are observed in the second and fourth quadrants for $K_{2} / K_{1}<9$ for positive $K_{1}$ only, which become more pronounced for higher absolute values of the ratio. This behavior is similar to that of the uniaxial anisotropy case. ${ }^{25}$

As follows, the dependencies of the remanent magnetization normalized to $M_{s}$, the normalized coercivity $h_{c}$ $\left[=H_{c} /\left(2\left|K_{1}\right| / M_{s}\right)\right]$, and remanence coercivity $h_{r}$ [ $\left.=H_{r} /\left(2\left|K_{1}\right| / M_{s}\right)\right]$ on the $K_{2} /\left|K_{1}\right|$ ratio are discussed in detail for negative and positive $K_{1}$ when $K_{2}$ $\in\left(-12 K_{1}, 6 K_{1}\right) ; H_{c}$ is the coercivity, and $H_{r}$ is the coercive field of the $M_{d}(H)$ curve $\left[M_{d}\left(H_{r}\right)=0\right]$.

\section{B. Saturation remanence}

When $K_{1}<0$, as can be seen from Fig. 2(a), $M_{r} / M_{s}$ is equal to the Gans' value, ${ }^{4} 0.8660$ for $K_{2} /\left|K_{1}\right| \in(-\infty, 2)$, and equals 0.9123 for $K_{2} /\left|K_{1}\right| \in(3, \infty)$. these $K_{2} /\left|K_{1}\right|$ values for the boundaries of the above two regions can be analytically determined by equating to zero the first and second partial derivatives of $E_{a}$ with respect to $\gamma$. When $K_{2} /\left|K_{1}\right|$ is raised starting from 2 , local minima along the $\langle 110\rangle$ directions appear, thus increasing the number of the possible remanence directions; as consequence, the remanent magnetization is increased, reaching its maximum of 0.9305 for $K_{2} /\left|K_{1}\right|$ $\approx 2.22$. A further increase of $K_{2} /\left|K_{1}\right|$ leads to a decrease of the remanence because the minima along the four $\langle 111\rangle$ directions become more shallow; these minima vanish for $K_{2} /\left|K_{1}\right|>3$. For larger $K_{2} /\left|K_{1}\right|$ values there are 12 easiest magnetization orientations along the face diagonals, $\langle 110\rangle$. For this case, it is possible to analytically determine the remanence magnetization value by using the procedure described below.

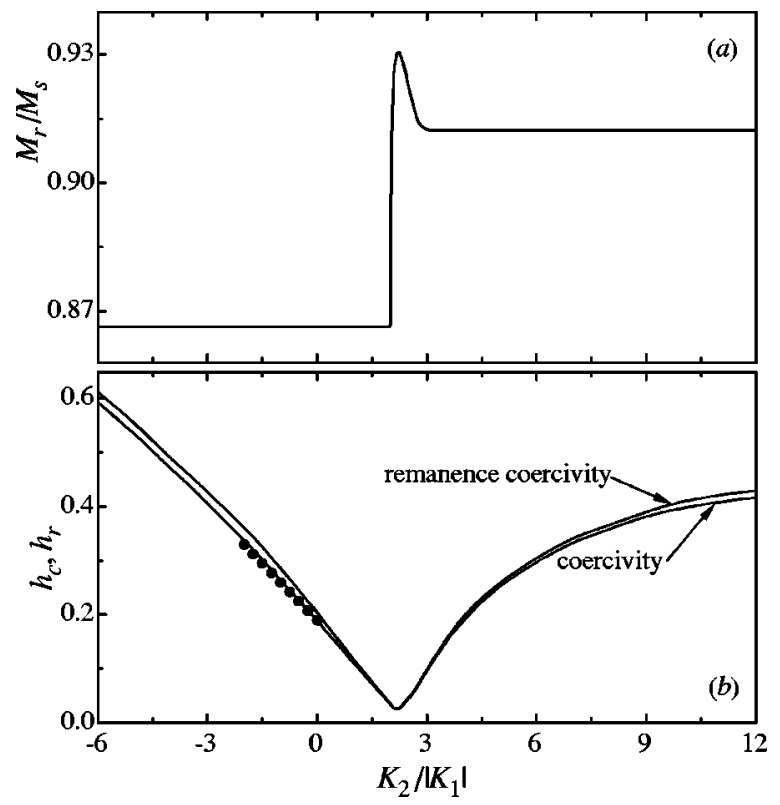

FIG. 2. Normalized saturation remanence $M_{r} / M_{s}$ (a), and the normalized to $2\left|K_{1}\right| / M_{s}$ coercivity $h_{c}$ and remanence coercivity $h_{r}$ (b) vs $K_{2} /\left|K_{1}\right|$ for negative $K_{1}$. The full circles in (b) represent the numerical results obtained by Usov and Peschany.

After applying and subsequently removing a sufficiently strong magnetic field, the magnetizations stay along the $\langle 110\rangle$ direction which is closest to the positive field direction. As long as the field direction is in the spherical triangle determined by the [101], [111], and [001] directions in Fig. 3, the magnetizations are expected to turn back to the [101] direction. If $\phi$ is the angle between $\mathbf{H}$ and the easiest direction, then the average remanence magnetization is

$$
\begin{aligned}
\frac{M_{r}}{M_{s}}=\overline{\cos \phi}=\int_{0}^{\pi / 4} \int_{0}^{\cot ^{-1} \cos \psi} \cos \phi \sin \theta d \theta d \psi / \\
\int_{0}^{\pi / 4} \int_{0}^{\cot ^{-1} \cos \psi} \sin \theta d \theta d \psi .
\end{aligned}
$$

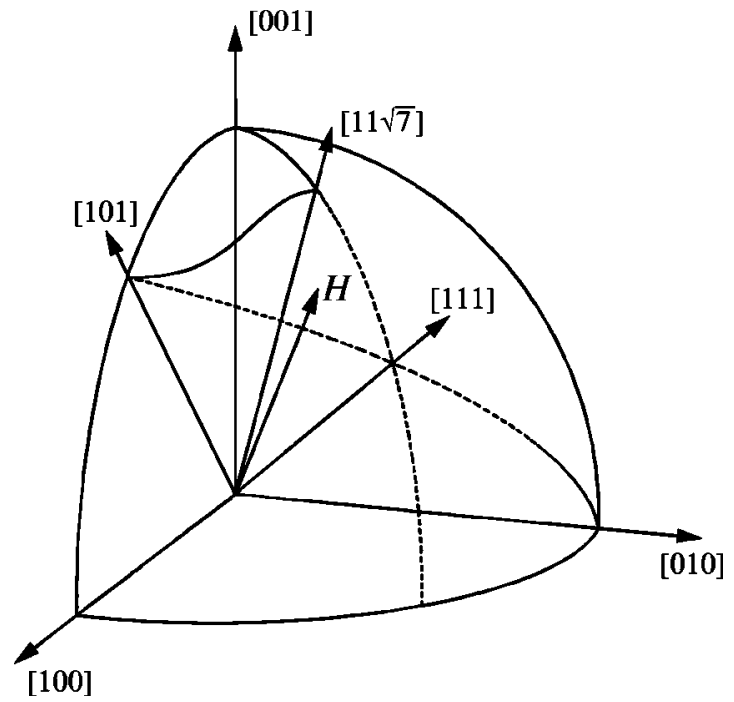

FIG. 3. The range of integration of the integral for $M_{r} / M_{s}$. 


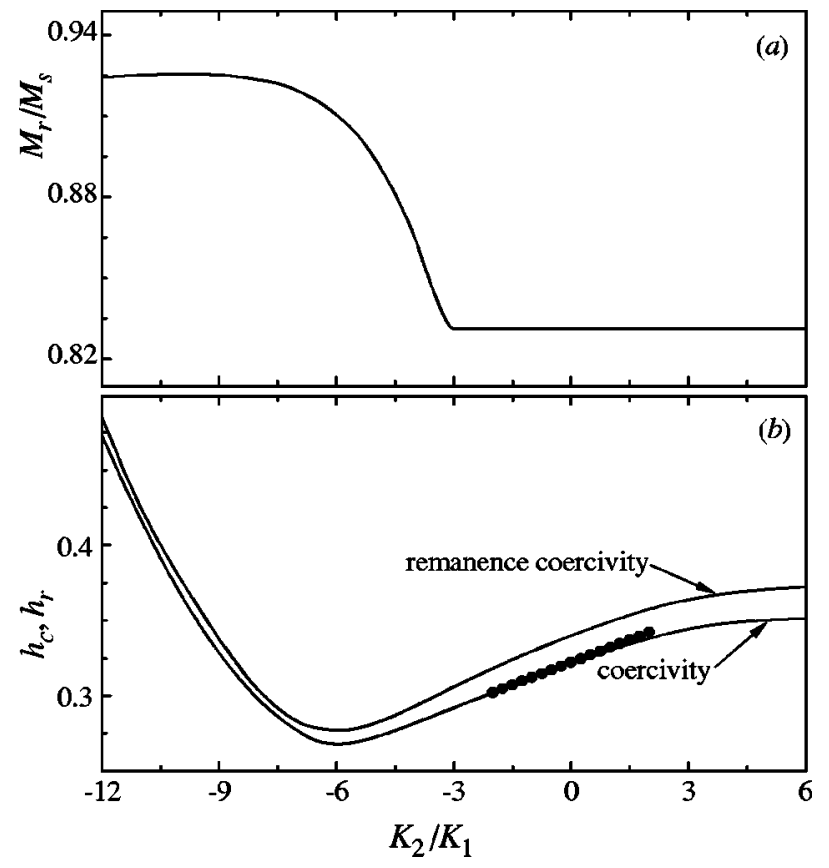

FIG. 4. Normalized saturation remanence $M_{r} / M_{s}$ (a), and the normalized to $2\left|K_{1}\right| / M_{s}$ coercivity $h_{c}$ and remanence coercivity $h_{r}$ (b) as a function of $K_{2} /\left|K_{1}\right|$ for positive $K_{1}$; the full circles in (b) represent the numerical results obtained by Usov and Peschany.

In the present case the easiest direction is [101], i.e., $\cos \phi$ $=1 / \sqrt{2}(\cos \psi \sin \theta+\cos \theta)$, the denominator in the above equation equals $4 \pi / 48$, thus

$$
\begin{aligned}
M_{r}= & \frac{48 M_{s}}{4 \pi} \int_{0}^{\pi / 4} \int_{0}^{\cot ^{-1} \cos \psi} \frac{1}{\sqrt{2}}(\cos \psi \sin \theta \\
& +\cos \theta) \sin \theta d \theta d \psi=0.9123 M_{s} .
\end{aligned}
$$

We should emphasize that the numerically calculated remanence coincides with the accuracy of $3 \times 10^{-5}$ with the analytically determined value.

The $M_{r} / M_{s}$ vs $K_{2} / K_{1}$ dependence for the case of positive $K_{1}$ is shown in Fig. 4(a). For $K_{2} / K_{1} \in(-3, \infty)$, there are six equivalent easiest magnetization orientations, and the saturation remanence is a constant, equal to the Gans' value of 0.8312 . The left boundary of this region is easy to be determined by equating to zero the first and second partial derivatives of $E_{a}$ to $\gamma$, and eliminating $\gamma$ for $\vartheta=\pi / 4$. Decreasing $K_{2} / K_{1}$ from -3 , eight additional minima along the $\langle 111\rangle$ directions appear and $M_{r} / M_{s}$ increases. It reaches a maximum of 0.926 for $K_{2} / K_{1} \approx-10$, and not at the spinreorientation point, i.e., $K_{2} / K_{1}=-9$, where there are seven equivalent easiest magnetization axes along the $\langle 100\rangle$ and $\langle 111\rangle$ directions; the easiest directions change from $\langle 100\rangle$ to $\langle 111\rangle$ for lower $K_{2} / K_{1}$ values.

A subsequent decrease of $K_{2} / K_{1}$ causes a gradual decrease of the remanence, which reaches 0.8660 for $K_{2} / K_{1}$ $=-\infty$. The reason is that for positive $K_{1}$, contrary to the negative $K_{1}$ case [when additional local minima exist only for $K_{2} /\left|K_{1}\right|$ in the $(2,3)$ interval], the local minima along the $\langle 100\rangle$ directions do not disappear for finite negative $K_{2} / K_{1}$ values, but only become more shallow with the ratio's decrease.
The above described procedure can be used to calculate the $M_{r} / M_{s}$ for the $K_{2} /\left|K_{1}\right|$ values at the spin-reorientation points. In order to determine the corresponding range of integration for positive $K_{1}$ and $K_{2}=-9 K_{1}$, for example, one can find the relation between $\psi$ and $\theta$ corresponding to the local energy maxima. The result is the solid curve connecting the points of intersection of [101] and $[11 \sqrt{7}]$ directions with the unit sphere in Fig. 3, i.e., $\psi$ $=\frac{1}{2} \sin ^{-1} \sqrt{\left(8-4 \csc ^{2} \theta\right) /\left(27 \sin ^{2} \theta-16\right)}$. Using this relation it is not difficult to calculate the average remanent magnetizations for both cases, [100] or [111] being the easiest axes, as well as their weighted average, which one could expect to be the remanence. This analytically calculated value is 0.9122 , which is slightly lower than the numerically obtained one, i.e., 0.9254 . The reason is that the seven easiest axes are not regularly distributed in the space, the symmetry of the energy surface is lower than that for only one type of easiest axes and, as a consequence, the remanent magnetization contributions for some field configurations are history dependent. If, for example, the saturation field is applied along the direction given by $\psi=0^{\circ}$ and $\theta=40^{\circ}$, after continuous decrease of the field the magnetization vector turns back to the [111] direction and not to [100], as the above static calculation of the integral assumes. In the numerical calculations, however, this magnetization follows the lowest energy path during the field's sweep like in real systems. Thus when more than one type of easiest directions coexist in a cubic anisotropy system, the above analytical procedure cannot be applied. In the present work the remanent magnetization is calculated numerically for these cases.

The lower energy symmetry and the history dependent remanence explain the fact that, for both negative and positive $K_{1}, M_{r} / M_{s}$ is maximum for $K_{2} /\left|K_{1}\right|$ values different from those at the spin-reorientation points, for which the spatially distributed easiest directions are equivalent (which only means that long these directions, the energy is minimum and has the same value).

Figure 5 shows the dependence of $M_{r} / M_{s}$ on $n$, the number of spatially distributed equivalent easiest axes. The remanent magnetization values for the cases of 1, 2, 3, and 4 easiest magnetization axes are taken from the literature, and the last three points correspond to the present results. All these values, as well as the corresponding coercivities, are given in Table II. In order to complete the table, the case of two easiest axes has also been considered; the anisotropy energy used and the corresponding results are given in the Appendix.

\section{Coercivity and remanence coercivity}

The dependencies of the normalized to $2\left|K_{1}\right| / M_{s}$ coercivity $h_{c}$ and remanence coercivity $h_{r}$ on $K_{2} /\left|K_{1}\right|$ are shown in Figs. 2(b) and 4(b) for positive and negative $K_{1}$, respectively, when $K_{2} \in\left(-12 K_{1}, 6 K_{1}\right)$. One can see that in this region all of the curves show only one minimum in the vicinity of the spin-reorientation point; the variation with the $K_{2} /\left|K_{1}\right|$ is stronger for the case of negative $K_{1}$, and the increase of the coercivity is more pronounced for $K_{2} /\left|K_{1}\right|$ 


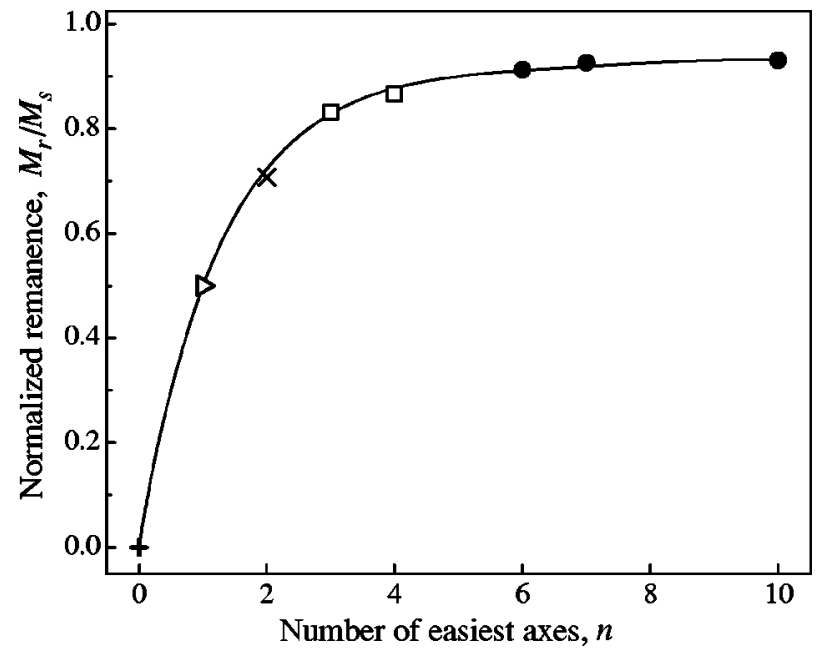

FIG. 5. Normalized saturation remanence $M_{r} / M_{s}$ vs $n$, number of spatially distributed equivalent easiest axes. The + symbol corresponds to superparamagnetic particles; $\triangleright$ is for the Stoner-Wohlfarth case; and $\times$ and $\square$ give the Wohlfarth and Tonge and the Gans' values, respectively. The last three points, the symbols - , correspond to the present results. The line is only a guide to the eye.

values lower than the spin-reorientation point value. For both positive and negative $K_{1}$, the $h_{c}$ and $h_{r}$ values are very close.

The coercivity values for certain regions are compared with the ones calculated by means of direct solution of the Landau-Lifshitz-Gilbert equation performed by Usov and Peschany, ${ }^{7}$ represented by the full circles in Figs. 2(b) and 4(b). A very good agreement between our and their calculations is observed.

As discussed above, out of the $K_{2} /\left|K_{1}\right|$ intervals shown in Figs. 2(a) and 4(a), the remanence does not show any peculiarities. The coercivity, however, shows more complex behavior. $h_{c}$ and $H_{c} / H_{a}$ as a function of $K_{2} /\left|K_{1}\right|$ are plotted in Fig. 6 for $K_{2} /\left|K_{1}\right| \in(-15,50)$; the expressions for the anisotropy field ${ }^{26} H_{a}$ are given in Table I. Only the $h_{c}$ curve for positive $K_{1}$ represents an additional feature not mentioned above: it has a local maximum of $h_{c}=0.352$ for $K_{2} / K_{1} \approx 9$, followed by a very slow decrease for further increase of $K_{2} / K_{1}$.

The $H_{c} / H_{a}$ dependencies are rather different from the $h_{c}$ ones. For $K_{1}<0$, the $H_{c} / H_{a}$ curve reaches its maximum for $K_{2} /\left|K_{1}\right| \approx-3.5$, has a very sharp local minimum for

TABLE II. Normalized remanent magnetization and coercivity for a different number of spatially distributed easiest axes, $n$.

\begin{tabular}{rccc}
\hline \hline$n$ & $M_{r} / M_{s}$ & $H_{c} /\left(2\left|K_{1}\right| / M_{s}\right)$ & $H_{c} / H_{a}$ \\
\hline 1 & $0.5000^{\mathrm{a}}$ & $0.479^{\mathrm{a}}$ & $0.479^{\mathrm{a}}$ \\
2 & $0.7071^{\mathrm{b}}$ & $>0.416$ & $<0.304$ \\
3 & $0.8312^{\mathrm{c}}$ & $>0.292$ & $>0.292$ \\
4 & $0.8660^{\mathrm{c}}$ & $>0.034$ & $>0.153$ \\
6 & 0.9123 & $>0.097$ & $<0.194$ \\
7 & 0.9254 & 0.331 & 0.248 \\
10 & 0.9303 & 0.027 & 0.159 \\
\hline \hline
\end{tabular}

${ }^{\text {aReference } 1 .}$

${ }^{\mathrm{b}}$ Reference 14 .

${ }^{\mathrm{c}}$ Reference 4.

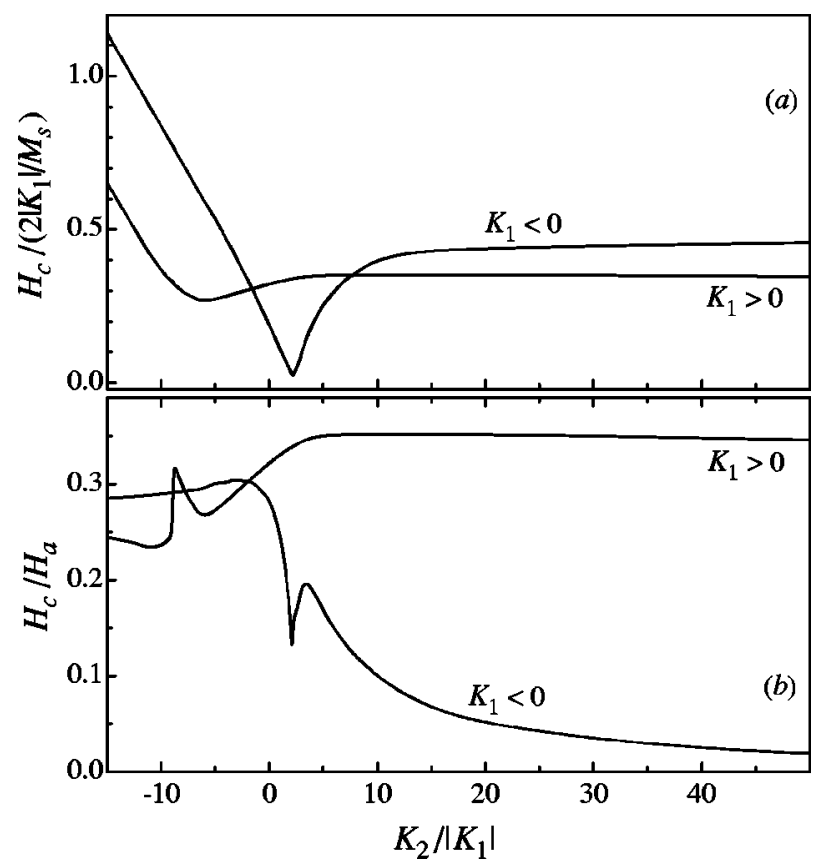

FIG. 6. Coercivity vs $K_{2} /\left|K_{1}\right|$ for both negative and positive $K_{1}$; (a) $H_{c} /\left(2\left|K_{1}\right| / M_{s}\right)$ and (b) $H_{c} / H_{a}$.

$K_{2} /\left|K_{1}\right|$ in the vicinity of the spin-reorientation point value, shows a local maximum for $K_{2} /\left|K_{1}\right| \approx 3.5$, and gradually decreases with the subsequent increase of $K_{2} /\left|K_{1}\right|$. The $H_{c} / H_{a}$ curve for positive $K_{1}$, when increasing $K_{2} / K_{1}$, has a minimum for $K_{2} / K_{1} \approx-11$ followed by a local maximum in the vicinity of the spin-reorientation point. There is another local minimum for $K_{2} / K_{1} \approx-6$, followed by the maximum for $K_{2} / K_{1} \approx 9$; for higher $K_{2} / K_{1}$ the anisotropy field equals $2 K_{1} / M_{s}$, and the curve is identical with the corresponding $h_{c}$ curve shown in Fig. 6(a).

One can see that for both positive and negative $K_{1}$ the variation in the $H_{c} / H_{a}$ curves is approximately three times smaller than that in the $h_{c}$ curves.

A parameter, derived from the hysteresis and $M_{d}(H)$ curves, is sometimes used for estimation the interparticle interaction effects, i.e., the so-called coercivity factor ${ }^{27}(\mathrm{CF})$, defined as $\mathrm{CF}=\left(H_{r} / H_{c}-1\right) \times 100$. For noninteracting fineparticle systems with uniaxial anisotropy ${ }^{27} \mathrm{CF} \approx 9 \%$, and for cubic anisotropy with $K_{2}=0$, the corresponding values are ${ }^{10}$ $\mathrm{CF} \approx 4 \%$ for $K_{1}<0$ and $\mathrm{CF} \approx 6 \%$ for $K_{1}>0$. Deviations of experimentally obtained $\mathrm{CF}$ values from the above theoretical ones are usually attributed to interparticle interactions. When the anisotropy is made up of a cubic magnetocrystalline and uniaxial components, rather high $\mathrm{CF}$ values have been calculated for some uniaxial anisotropy directions for dominant uniaxial anisotropy. ${ }^{17}$ This means that even for effectively "uniaxial" particles, the CF values can be much higher than those for pure uniaxial or cubic anisotropy.

For the cases considered in the present work, due to the very small difference between the $h_{c}$ and $h_{r}$ values for both positive and negative $K_{1}$, the CF does not overcome $9 \%$.

\section{Remanent magnetization plots}

A technique based on a comparison of the above mentioned remanence curves has been extensively used for esti- 


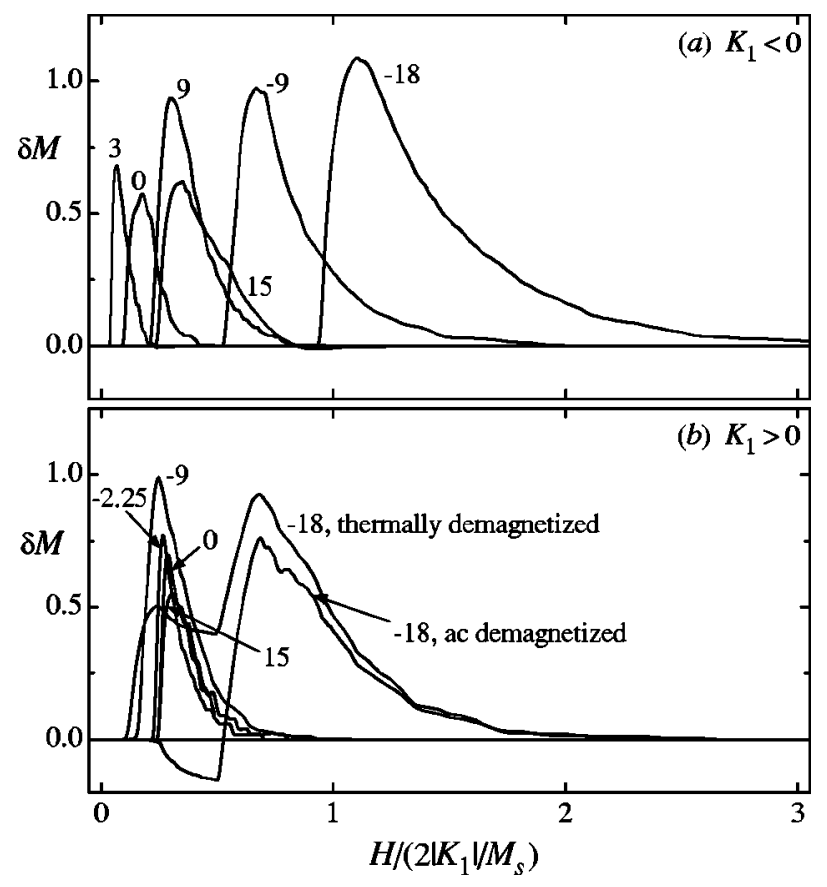

FIG. 7. Representative $\delta M$ plots for several $K_{2} /\left|K_{1}\right|$ values for the cases of negative (a) and positive (b) $K_{1}$. The numbers denote the $K_{2} /\left|K_{1}\right|$ ratios.

mation of interaction effects in fine-particle systems in the last decade. For noninteracting single-domain uniaxial anisotropy particles, these curves are connected via the Wohlfarth relation ${ }^{24} M_{d}(H)=1-2 M_{r}(H)$. Henkel ${ }^{28}$ first noted that the experimental variation of $M_{d}(H)$ with $M_{r}(H)$ for heterogeneous alloys gave plots showing both positive and negative curvature. Kelly et al. ${ }^{29}$ have used the remanence curves to estimate interactions in granular thin films, due to the so-called $\delta M$ plot,

$$
\delta M(H)=M_{d}(H)-1+2 M_{r}(H) .
$$

Usually, negative $\delta M$ values are taken to indicate "demagnetizing" interaction, i.e., interactions that tend to stabilize the demagnetized state; positive values are attributed to interactions promoting the magnetized state. This is true, however, for the uniaxial anisotropy case only. For the case of pure cubic anisotropy, for both positive and negative $K_{1}$ (and $K_{2}=0$ ), the $\delta M$ plots for the noninteracting case are positive. ${ }^{10}$ When cubic and uniaxial components coexist, the corresponding $\delta M$ may show a great variety of shapes when the uniaxial anisotropy does not dominate. ${ }^{17}$ Also, the $M_{r}(H)$ curve depends on the method by which the demagnetized state is produced. From dc, ac, or thermal demagnetization one can obtain very different initial magnetization and $M_{r}(H)$ curves, and the corresponding $\delta M$ plots can differ significantly.

In the present study remanence curves and the corresponding $\delta M$ plots have been calculated for various $K_{2} /\left|K_{1}\right|$ ratios. Some representative $\delta M$ plots obtained after thermal demagnetization are shown in Fig. 7 for negative and positive $K_{1}$. For the cases when there are two types of energy minima, i.e., $K_{2} /\left|K_{1}\right|$ is in the vicinity of the spinreorientation points, the $M_{r}(H)$ curves and subsequently the $\delta M$ plots, obtained after ac and thermal demagnetized states, are different. Curves obtained after dc demagnetization have not been considered.

When $K_{1}<0$ the $\delta M$ plots have, in practice, positive values only. The exceptions are the plots for positive $K_{2} /\left|K_{1}\right|$, but the negative values in these curves are very small as compared to the positive ones.

For $K_{1}>0$ there is a very small variation of the $\delta M$ plots for $K_{2} / K_{1}>-9$; for sufficiently high absolute values (and negative) $K_{2} / K_{1}$, the $\delta M$ plots obtained after ac demagnetization differ from the ones obtained after thermal demagnetization, showing negative values for small $h$. The $\delta M$ plot for positive $K_{1}$ and $K_{2} / K_{1}=-18$, obtained after ac demagnetization, is shown in Fig. 7(b) as well.

\section{COMPARISON WITH THE EXPERIMENT}

The predicted remanence behavior is compared with the one of a $\mathrm{Tb}_{0.6} \mathrm{Ho}_{0.4} \mathrm{Fe}_{2}$ sample, a rare-earth iron Laves compound representing spin-reorientation transition of the easiest direction of magnetization ${ }^{19-21}$ from $\langle 110\rangle$ to $\langle 111\rangle$ in the vicinity of $85 \mathrm{~K}$.

A bulk $\mathrm{Tb}_{0.6} \mathrm{Ho}_{0.4} \mathrm{Fe}_{2}$ alloy button was prepared by arcmelting of the starting materials under an argon atmosphere. The button was remelted several times and vacuum annealed for 3 weeks at $1050^{\circ} \mathrm{C}$ in order to produce a single-phase alloy. Subsequently, it was powderized by ball-milling for 5 $\mathrm{h}$ in cyclohexane, using $7 \mathrm{~mm}$ diameter agate balls in a quartz cylinder and a roller mill.

The structural characterization was performed via conventional X-ray powder diffraction performed on a Philips X'Pert Materials Research Diffractometer (MRD) machine employing $\mathrm{Cu} K \alpha$ radiation. The analysis of the bulk material indicates a cubic crystal structure. The diffraction pattern for the milled sample is practically identical with the one of the bulk alloy. No traces of extra phases, including oxides, are observed.

The magnetization data were acquired for this nonorientated fine powder sample by a superconducting quantum interference device (SQUID) magnetometer with a maximum applied field of $50 \mathrm{kOe}$. Hysteresis loops were measured at two temperatures, 5 and $300 \mathrm{~K}$. These loops are shown in Fig. 8 and it can be seen that their shape is characteristic of a sample consisting of two magnetic phases: very small particles that exhibit superparamagnetism (at sufficiently high temperature, the magnetization vectors of these particles are thermally agitated over their potential barriers, permitting them to rotate around their equilibrium directions) responsible for the slope of the high field magnetization, and larger, ferromagnetic particles, which account for the hysteresis. Both loops are closed at fields rather smaller than the maximum applied field, which means that the anisotropy field of the ferromagnetic phase is lower than the maximum applied field for the whole temperature interval.

In Fig. 9 the saturation remanent magnetization $M_{r}$, the magnetization at $50 \mathrm{kOe}$, and the zero-field cooled magnetization at an applied field of $5 \mathrm{kOe}$ as a function of the temperature from 5 to $300 \mathrm{~K}$ for the $\mathrm{Tb}_{0.6} \mathrm{Ho}_{0.4} \mathrm{Fe}_{2}$ sample are plotted. For the saturation remanence vs temperature measurement, the sample was cooled from 300 to $10 \mathrm{~K}$ in a field 


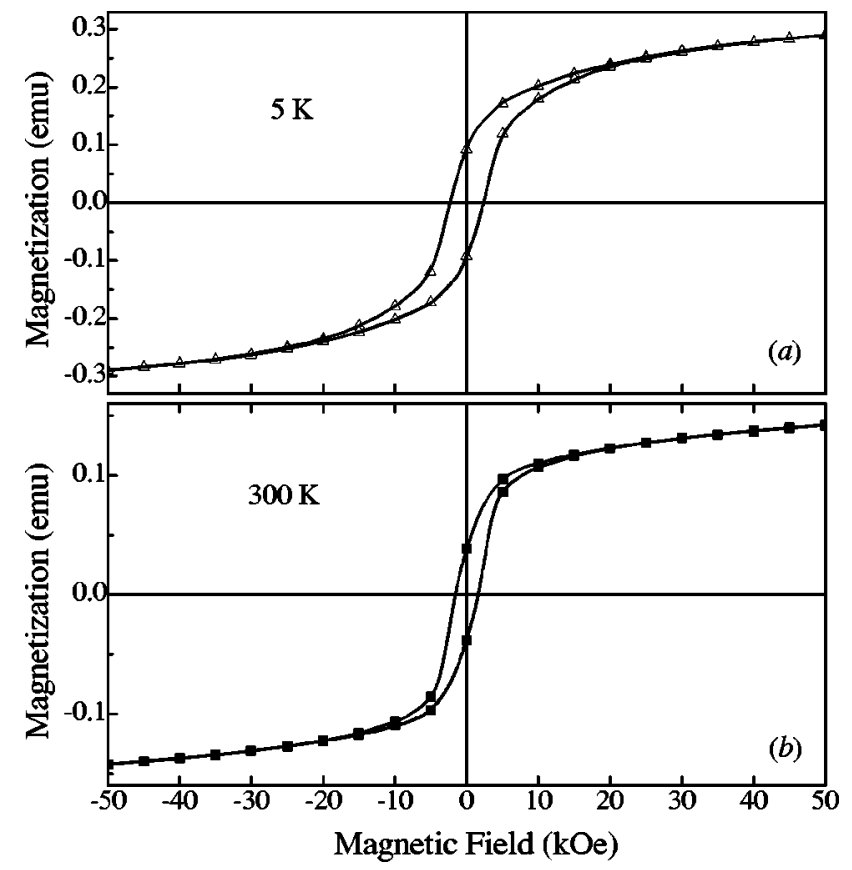

FIG. 8. Hysteresis loops for a $5 \mathrm{~h}$ ball-milled $\mathrm{Tb}_{0.6} \mathrm{Ho}_{0.4} \mathrm{Fe}_{2}$ sample at $5 \mathrm{~K}$ (a) and at room temperature (b).

of $50 \mathrm{kOe}$; at each measurement temperature the magnetization was measured, the field was removed, and $M_{r}$ was measured, then the field was applied again and the temperature was subsequently lowered. This procedure and the fact that the ferromagnetic phase is saturated at $300 \mathrm{~K}$ (closed hysteresis loop), assure that the curve in Fig. 9(a) represents the saturation remanence of the ferromagnetic part of the sample. It was not possible, however, to plot the $M_{r} / M_{s}$ curve for the whole temperature range; as seen in Fig. 9(b), $M(T)$ for $H=50 \mathrm{kOe}$ does not correspond to $M_{s}(T)$ due to the presence of the superparamagnetic (increasing with the temperature raise) phase.

The most interesting feature of Fig. 9 is the well expressed singularity in the $M_{r}$ vs $T$ dependence for $T \approx 85 \mathrm{~K}$. This is exactly the temperature for which the spinreorientation transition of the easiest directions of magnetization from $\langle 110\rangle$ to $\langle 111\rangle$ occurs for this compound when increasing $T$ according to the one-ion model theory predictions. ${ }^{19}$ The general trend to decrease of $M_{r}$ with the temperature raise is due to the superparamagnetic/ ferromagnetic phase ratio increase with $T$, which is supported by the rather fast but gradual decrease of the magnetization for $H=50 \mathrm{kOe}$ as seen in Fig. 9(b). To explain the singularity in the $M_{r}$ dependence, one should compare this curve with that shown in Fig. 2(a). One can do this comparison despite the fact that the ferromagnetic part of our sample could contain larger particles; the numerical $M_{r} / M_{s}$ values shown in Figs. 2(a) and 4(a) are valid for polycrystalline samples as well. ${ }^{4}$ The temperature increase is equivalent to a $K_{2} /\left|K_{1}\right|$ decrease when starting from values higher than 3; both plots show the same feature: a maximum in the vicinity of the $\langle 110\rangle$ to $\langle 111\rangle$ easiest direction transition. This spinreorientation transition at $T=85 \mathrm{~K}$ is also confirmed by the rather abrupt reduction of the magnetization in the $M(T)$

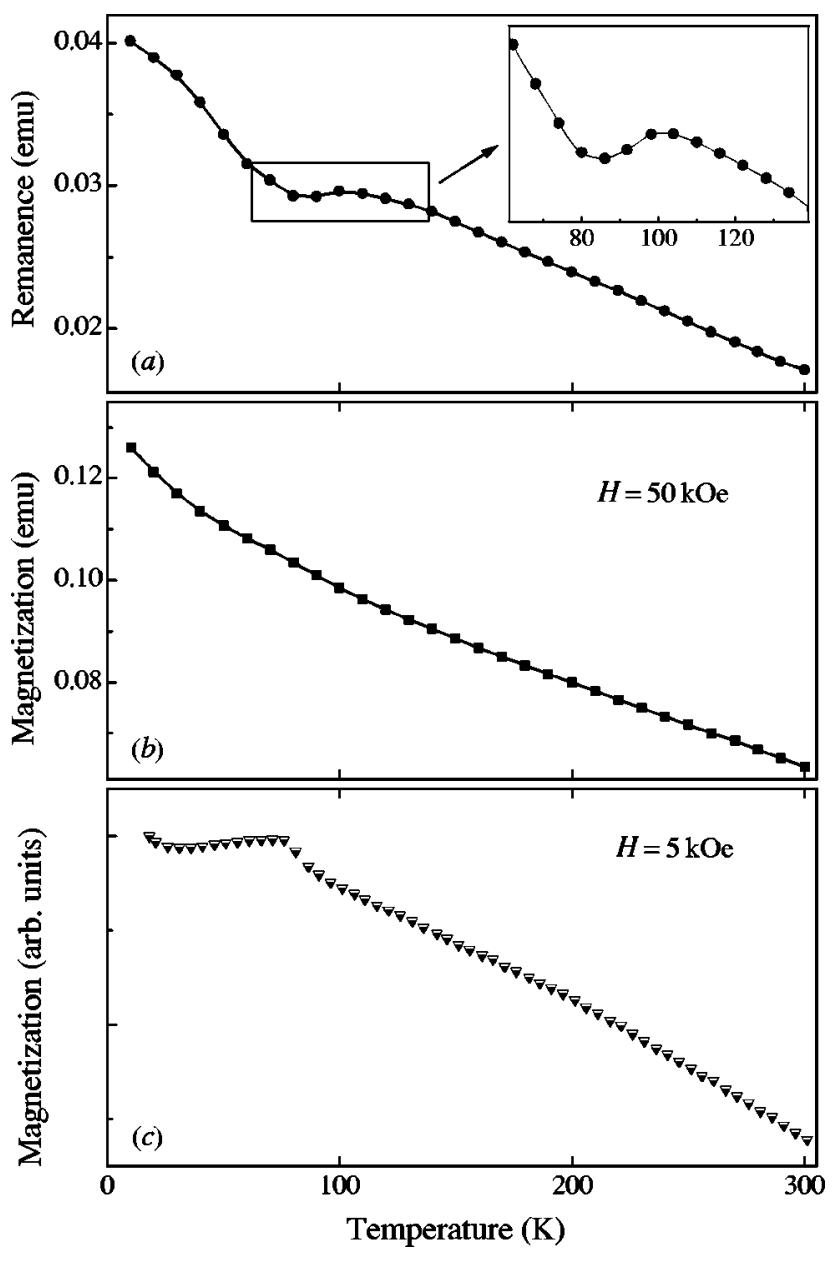

FIG. 9. (a) Remanence magnetization $M_{r}$, (b) magnetization $M$ at $H=50$ $\mathrm{kOe}$, and (c) zero-field cooled curve at $5 \mathrm{kOe}$ for a $\mathrm{Tb}_{0.6} \mathrm{Ho}_{0.4} \mathrm{Fe}_{2}$ sample as a function of the temperature.

dependence for $H=5$ kOe shown in Fig. 9(c). When $T$ is increased in this temperature range, the number of easiest axes is reduced from 6 to 4 . This leads, for the majority of the particles, to changes of their equilibrium states, and subsequently, to a decrease of their magnetization projections along the applied field direction.

\section{CONCLUSIONS}

The magnetization and remanent magnetization curves have been calculated for a disordered system of noninteracting single-domain particles with cubic anisotropy, taking into consideration the first two anisotropy constants; the calculations cover a much wider $K_{2}$ range than previous ones. The dependencies of the coercivity and the remanence coercivity on $K_{2}$ have been discussed. It has also been obtained that, due to the very small difference between the $h_{c}$ and $h_{r}$ values, the coercivity factor does not overcome $9 \%$.

$\delta M$ plots have been constructed for several representative $K_{2} /\left|K_{1}\right|$ ratios and it has been obtained that they are predominantly positive.

The calculated saturation remanence vs $K_{2} /\left|K_{1}\right|$ dependencies for both positive and negative $K_{1}$ show maxima in the vicinity of the spin-reorientation transitions of the easiest 


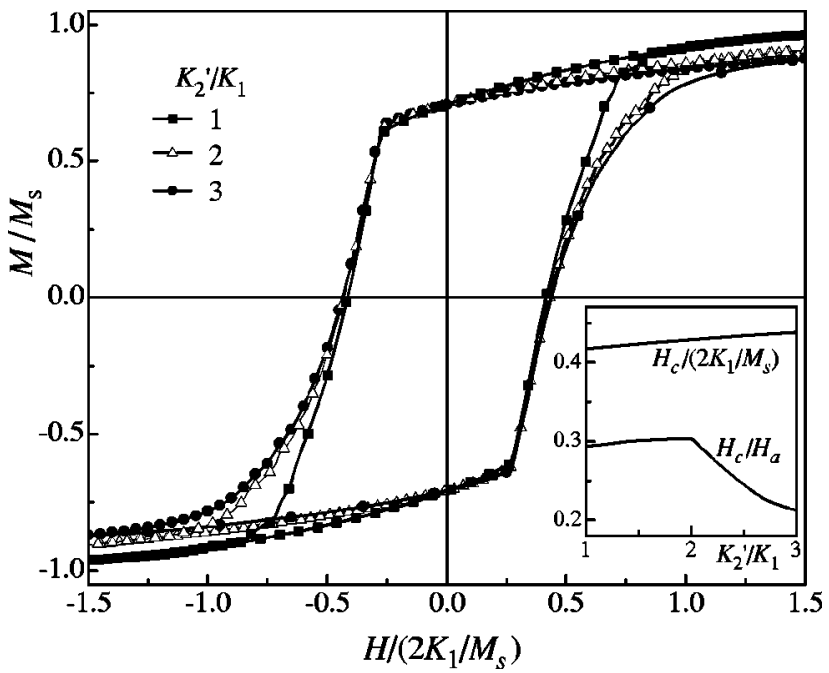

FIG. 10. Representative hysteresis loops calculated for a disordered system of noninteracting single-domain particles with biaxial anisotropy. The inset shows the dependence of the normalized coercivities on the $K_{2}^{\prime} / K_{1}$ ratio.

directions of magnetization. The $M_{r} / M_{s}$ for the case when the face diagonals $\langle 110\rangle$ are the easiest magnetization orientations has been analytically determined, i.e., $M_{r}$ $=0.9123 M_{s}$. It has been shown that when more than one type of easiest directions coexist, due to the history dependence of the remanent magnetization, $M_{r} / M_{s}$ can only be obtained numerically.

The predicted remanence behavior for systems representing spin-reorientation was compared with the one of a $\mathrm{Tb}_{0.6} \mathrm{Ho}_{0.4} \mathrm{Fe}_{2}$ powder sample, and an excellent agreement between numerical and experimental data in the spinreorientation transition region has been found.

\section{ACKNOWLEDGMENTS}

The authors thank Dr. J. Schaf for access to the SQUID magnetometer and for assistance with the measurements, and also Dr. E. Gattef and Dr. S. R. Teixeira for assistance with the x-ray characterization. This work has been supported by Conselho Nacional de Desenvolvimento Científico e Tecnológico (CNPq, Brazil), Fundação de Amparo à Pesquisa do Estado do Rio Grande do Sul (FAPERGS, Brazil), Financiadora de Estudos e Projetos (FINEP, Brazil), as well as by the Scientific Foundation of the Sofia University, Bulgaria.

\section{APPENDIX}

Wohlfarth and Tonge ${ }^{13}$ have shown that if $n$ equivalent easiest axes are distributed in a plane, the reduced remanent magnetization $M_{r} / M_{s}=\frac{1}{2} n \sin (\pi / 2 n)$. For $n=2$ this gives $1 / \sqrt{2}$ or approximately 0.7071 . In order to calculate the magnetization curves for a disordered system of noninteracting single-domain particles with such a biaxial anisotropy, we used the energy expression given by Eq. (1), where the second term was substituted by $K_{2}^{\prime} \alpha_{3}^{2}$, considering positive $K_{1}$ and $K_{2}^{\prime}$. The total free energy in units of $2 K_{1}$ becomes

$$
\eta=\frac{1}{8}\left(\sin ^{4} \gamma \sin ^{2} 2 \vartheta+\sin ^{2} 2 \gamma\right)+\frac{K_{2}^{\prime}}{2 K_{1}} \cos ^{2} \gamma-h \cos \varphi .
$$

If $K_{2}^{\prime} K_{1} \geqslant 1$, there are only four easiest orientations: [100], [010], [100], and [010], i.e., the two principal cube axes in the $X Y$ plane. The anisotropy field $H_{a}$ of the system equals $2 \sqrt{2} K_{1} / M_{s}$ for $K_{2}^{\prime} / K_{1} \in[1,2]$, and $2 \sqrt{6} / 9(1$ $\left.+K_{2}^{\prime} / K_{1}\right)^{3 / 2} K_{1} / M_{s}$ if $K_{2}^{\prime} / K_{1}>2$.

Hysteresis loops calculated for $K_{2}^{\prime} / K_{1}=1,2$, and 3 are shown in Fig. 10. The inset in the figure shows the dependence of the normalized coercivities on the $K_{2}^{\prime} / K_{1}$ ratio. It was obtained that $h_{c}=H_{c} /\left(2 K_{1} / M_{s}\right)=0.416$ for $K_{2}^{\prime} / K_{1}$ $=1$, and weakly and gradually increases when increasing $K_{2}^{\prime} / K_{1}$. The reduced to $H_{a}$ coercivity, however, increases starting from 0.294 for $K_{2}^{\prime} / K_{1}=1$, reaches its maximum of 0.304 for $K_{2}^{\prime} / K_{1}=2$, and then decreases gradually for further increase of the anisotropy constants ratio, reaching asymptotically 0 when $K_{2}^{\prime} / K_{1} \rightarrow \infty$.

The corresponding initial magnetization curves, remanence curves, $\delta M$ plots, and coercivity factors can be readily calculated.

${ }^{1}$ E. C. Stoner and E. P. Wohlfarth, Philos. Trans. R. Soc. London, Ser. A 240, 559 (1948)

${ }^{2}$ C. E. Johnson and W. F. Brown, J. Appl. Phys. 30, 320S (1961).

${ }^{3}$ E. W. Lee and J. E. L. Bishop, Proc. Phys. Soc. London 89, 661 (1966).

${ }^{4}$ R. Gans, Ann. Phys. (Leipzig) 15, 28 (1932).

${ }^{5}$ S. Kaya, Z. Phys. 84, 705 (1933).

${ }^{6}$ E. C. Stoner and P. Rhodes, Philos. Mag. 7, 481 (1949).

${ }^{7}$ N. A. Usov and S. E. Peschany, J. Magn. Magn. Mater. 174, 247 (1997).

${ }^{8}$ I. Joffe and R. Heuberger, Philos. Mag. 2, 1051 (1974).

${ }^{9}$ J. Geshev, O. Popov, V. Masheva, and M. Mikhov, J. Magn. Magn. Mater. 92, 185 (1990).

${ }^{10}$ J. Geshev and M. Mikhov, J. Magn. Magn. Mater. 104-107, 1569 (1992).

${ }^{11}$ M. Walker, P. I. Mayo, K. O'Grady, S. W. Charles, and R. W. Chantrell, J. Phys.: Condens. Matter 5, 2779 (1993).

${ }^{12}$ M. Walker, P. I. Mayo, K. O'Grady, S. W. Charles, and R. W. Chantrell, J. Phys.: Condens. Matter 5, 2793 (1993).

${ }^{13}$ E. P. Wohlfarth and D. G. Tonge, Philos. Mag. 2, 1333 (1957).

${ }^{14}$ D. G. Tonge and E. P. Wohlfarth, Philos. Mag. 3, 536 (1958).

${ }^{15}$ A. S. Arrott, Nanomagnetism, edited by A. Hernando (Kluwer, Dordrecht, 1993), p. 73.

${ }^{16}$ J. Geshev, A. D. C. Viegas, and J. E. Schmidt, J. Appl. Phys. 84, 1488 (1998).

${ }^{17}$ J. Geshev, M. Mikhov, and J. E. Schmidt, J. Appl. Phys. 88, 7321 (1999).

${ }^{18}$ R. F. Pearson, J. Appl. Phys. 33, 1236 (1962).

${ }^{19}$ U. Atzmony, M. P. Dariel, E. R. Bauminger, D. Lebenbaum, I. Nowik, and S. Ofer, Phys. Rev. Lett. 28, 244 (1972).

${ }^{20}$ U. Atzmony, M. P. Dariel, E. R. Bauminger, D. Lebenbaum, I. Nowik, and S. Ofer, Phys. Rev. B 9, 4220 (1973).

${ }^{21}$ C. M. Williams and N. C. Koon, Phys. Rev. B 11, 4360 (1975).

${ }^{22}$ J. García-Otero, M. Porto, and J. Rivas, J. Appl. Phys. 87, 7376 (2000).

${ }^{23}$ R. M. Bozorth, Phys. Rev. 50, 1076 (1936).

${ }^{24}$ E. P. Wohlfarth, J. Appl. Phys. 29, 595 (1958).

${ }^{25}$ V. Masheva, J. Geshev, and M. Mikhov, J. Magn. Magn. Mater. 140-144, 371 (1995).

${ }^{26}$ R. F. Pearson, Experimental Magnetism, edited by G. M. Kalvius and R. S. Teblle (Wiley, Chichester, 1979), p. 138.

${ }^{27}$ A. R. Corradi and E. P. Wohlfarth, IEEE Trans. Magn. 14, 861 (1978).

${ }^{28}$ O. Henkel, Phys. Status Solidi 7, 919 (1964).

${ }^{29}$ P. E. Kelly, K. O'Grady, P. I. Mayo, and R. W. Chantrell, IEEE Trans. Magn. 2, 3881 (1989). 